\title{
Optical image coding with a circular Dammann grating
}

\author{
Kyu B. Doh, ${ }^{1}$ Kelly Dobson, ${ }^{2}$ Ting-Chung Poon, ${ }^{2, *}$ and Po Sheun Chung ${ }^{3}$ \\ ${ }^{1} S c h o o l$ of Electronics and Telecommunication Engineering, Korea Aerospace University, \\ 100 Hanggongdae-gil, Goyang-City, 412-791, Korea \\ ${ }^{2}$ Bradley Department of Electrical and Computer Engineering, Virginia Tech, Blacksburg, Virginia 24061, USA \\ ${ }^{3}$ Department of Electronic Engineering, City University of Hong Kong, Tat Chee Avenue, Kowloon, Hong Kong \\ *Corresponding author: tcpoon@vt.edu
}

Received 2 July 2008; revised 11 November 2008; accepted 18 November 2008; posted 19 November 2008 (Doc. ID 98257); published 22 December 2008

\begin{abstract}
A circular Dammann grating in the front focal plane of a lens is used to generate an annular beam in the back focal plane to code an object by two-dimensional scanning. The coded image of the object is decoded digitally by convolving it with the same annular beam used to code the object. Simple inverse filtering is subsequently used to improve the contrast of the decoded image. (C) 2008 Optical Society of America OCIS codes: $\quad 070.0070,100.0100,070.4560$.
\end{abstract}

\section{Introduction}

Circular Dammann gratings (CDG) were first proposed by Zhou et al. in 2003 [1]. The CDG might find many interesting applications, such as measuring angular rotation of mirrors [2] and generating disk-shaped distributed feedback lasers [3], where circular gratings have been used. To the best of our knowledge, actual applications to CDGs have been limited only to focal-length measurements [4]. In this paper we discuss the use of CDGs for optical image coding. In Section 2 we describe the coding and decoding scheme and demonstrate the idea by computer simulations. In Section 3 we describe an optical scanning system that will be used for optical coding. In Section 4 we discuss the circular grating and some of its properties. We then show experimental results in Section 5 . In Section 6 we perform simple inverse filtering on the decoded image to enhance the quality of the image. Finally, in Section $\underline{7}$, we make some concluding remarks.

\section{Coding and Decoding Scheme}

Image coding has a long history, especially in holography and $\mathrm{x}$-ray tomographic imaging [믈. The re-

0003-6935/09/010134-06\$15.00/0

() 2009 Optical Society of America construction of coded images is usually accomplished digitally [8], but the task is often computationally demanding, as the coded image has a much larger space-bandwidth product than that of the data itself [9]. For this reason, optical reconstruction techniques have been investigated [10-13]. In this paper we investigate an optical scanning system for image coding. Decoding can be performed digitally or optically. Our scheme is to find a function that can be used for coding as well as decoding, which simplifies the procedures of coding and decoding. The coding and decoding scheme discussed here is reminiscent of coded aperture imaging already mentioned [ $\underline{6}-\underline{13}]$. In general, the apertures are selected to have some desirable autocorrelation or cross-correlation properties such that the coded image can be decoded to extract the original image. While a huge amount of work has been published with regard to digital image coding [14], research in optical image coding remains vibrant [15-20]. One of the reasons for using optical coding is that information, such as images, that needs to be coded already exists in the optical domain. Another reason is that optical coding, as opposed to electronic or digital coding, can provide many degrees of freedom for coding information. However, when the coded image is already in digital form, digital decoding is logical. Indeed what we are proposing here is a hybrid, i.e., optical/digital system 
that is more flexible than an all-optical or all-digital system alone.

Let us assume the coding function is $c(x, y)$. The original image is given by $o(x, y)$. Hence the coded image $i_{c}(x, y)$ is

$$
i_{c}(x, y)=o(x, y) * c(x, y),
$$

where $*$ stands for two-dimensional (2-D) convolution defined by

$f(x, y) * g(x, y)=\int_{-\infty}^{\infty} \int_{-\infty}^{\infty} f\left(x^{\prime}, y^{\prime}\right) g\left(x-x^{\prime}, y-y^{\prime}\right) \mathrm{d} x^{\prime} \mathrm{d} y^{\prime}$.

To decode the image, we can convolve the coded image with a decoding function $d(x, y)$ to give

$$
i_{d}(x, y)=i_{c}(x, y) * d(x, y) .
$$

Substituting Eq. (1) into Eq. (2)), we have

$$
i_{d}(x, y)=i_{c}(x, y) * d(x, y)=o(x, y) * c(x, y) * d(x, y) .
$$

Note that $c(x, y) * d(x, y)$ in Eq. (3) is the point spread function (psf) of the overall system. If $c(x, y)$ and $d(x, y)$ are suitably chosen such that the following condition applies:

$$
c(x, y) * d(x, y)=\delta(x, y),
$$

where $\delta(x, y)$ is a 2-D Delta function, then Eq. (3) becomes

$$
i_{d}(x, y)=o(x, y) * \delta(x, y)=o(x, y),
$$

and we have extracted the original image $o(x, y)$. Equation (4) is called a Delta reconstruction condition. The objective is to design the coding and decoding functions such that the condition in Eq. (4) is satisfied. We propose that an impulse ring for the coding and decoding functions will satisfy the Delta reconstruction condition approximately. We will then discuss an optical system that can implement the idea.

Figure 1(a) shows an impulse ring as a coding function, and Fig. 1(b) shows the convolution of the impulse ring by itself or autoconvolution of the impulse ring, where we have used the same ring as a decoding function. Note that the autoconvolution of the impulse ring has a strong peak at the origin (deltalike function) but has a faint circular "bump" away from the center. The circular bump has a radius that is twice as large as that of the impulse ring. In Fig. 1(c), we show the cross section of the autoconvolution of the impulse ring through its peak. Now if we can minimize the influence of the bump, we will come up with a good approximation to the condition set forth in Eq. (4). In the next example we show how the effect of the bump can be minimized by properly designing the size of the impulse

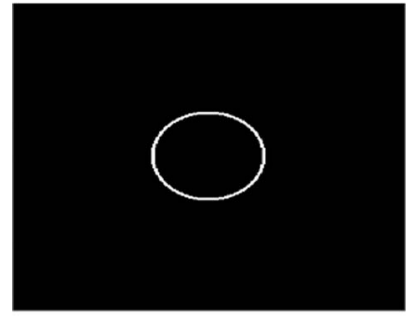

(a)

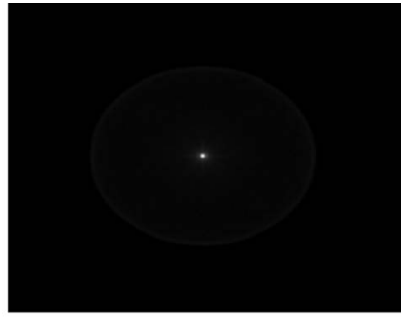

(b)

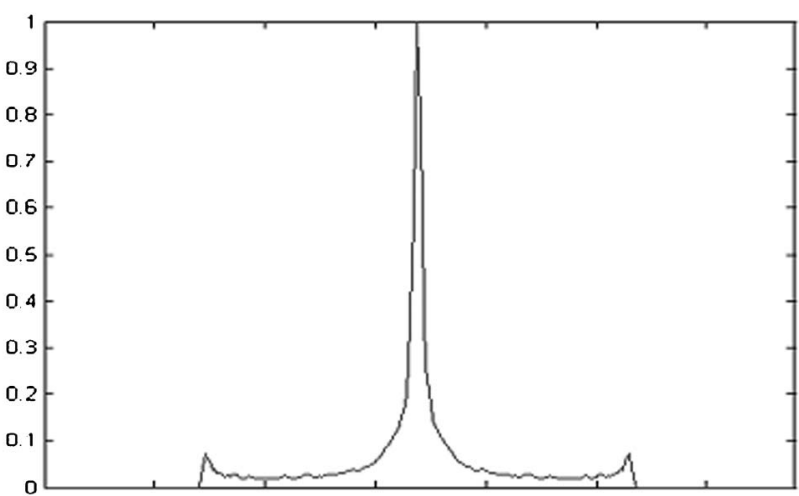

(c)

Fig. 1. (a) Impulse ring, (b) autoconvolution of the impulse ring, (c) cross section of (b) through the peak.

ring with respect to that of the image to be coded. Figure 2(a) shows the original "VT" image, Fig. 2(b) is the impulse ring to serve as the coding function, and Fig. 2(c) is the coded image. In Fig. 2(d) we show the decoded image as calculated according to Eq. (3). We see the image has been decoded correctly but with some annoying background. The annoying background is due to the circular bump. The effect of the bump can be minimized if we choose the size of the impulse ring to be larger than that of the image to be coded. This is illustrated in the series of figures shown in Fig. 3. Indeed in Fig. 3(d), we observe a clea-

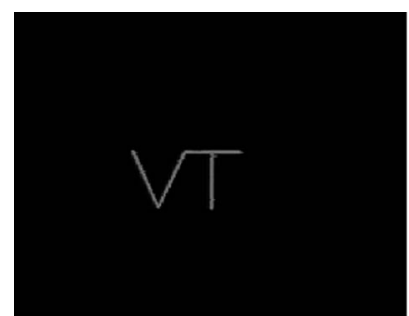

(a)

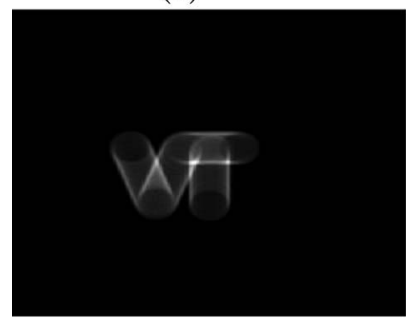

(c)

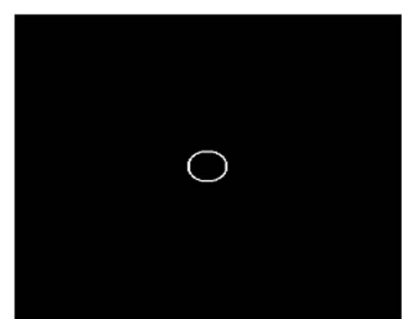

(b)

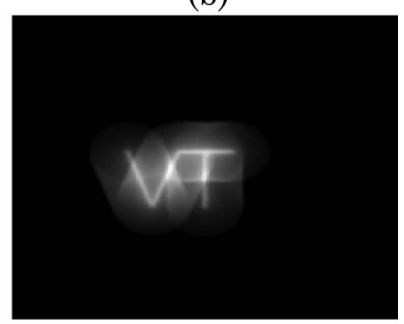

(d)
Fig. 2. (a) Original "VT" image, (b) impulse ring as a coding function, (c) coded image, (d) decoded image. 


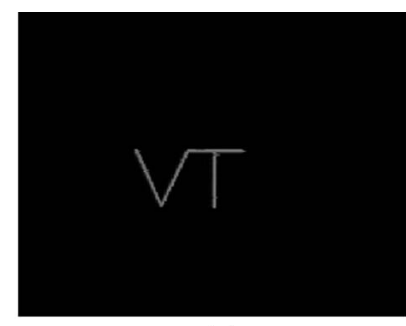

(a)

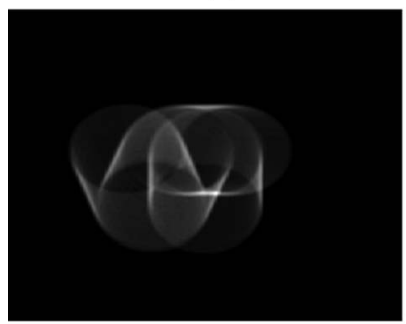

(c)

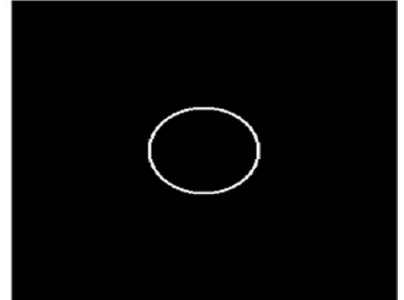

(b)

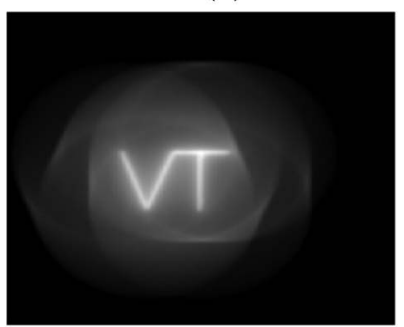

(d)
Fig. 3. (a) Original "VT" image, (b) impulse ring as a coding function, (c) coded image, (d) decoded image.

ner decoded image as compared to the decoded image shown in Fig. 2(d). We shall now turn our attention to see how the coding and decoding scheme can be implemented in an optical system in Section $\underline{3}$.

\section{Optical Scanning System}

We shall implement our coding/decoding scheme using an optical scanning system shown in Fig. 4 . Figure 4 shows an idealized version of a conventional laser-scanning image processor [21]. $p(x, y)$ is the pupil function located in the front focal plane (or the pupil plane) of lens $\mathrm{L}$ with focal length $f$. The pupil function, illuminated by a board laser, forms an intensity psf (ipsf), $\operatorname{ipsf}(x, y)$, onto the object $I_{0}(x, y)$ at the back focal plane of lens L. The ipsf is then two dimensionally scanned over the object by an $x-y$ scanner to give a scanned output image $I_{i}(x, y)$ to be displayed on the 2-D display. In Fig. 4, a photodetector accepts the light intensity being transmitted through the input transparency $I_{0}(x, y)$ and converts the light energy into a scanned electrical signal to be displayed on a 2-D display or stored in a computer for further processing.

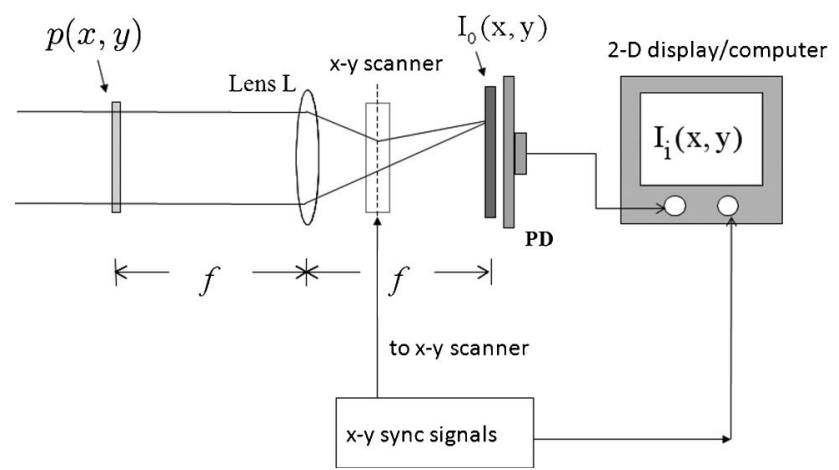

Fig. 4. Conventional laser-scanning image processor. PD, photodetector.
The image processor shown in Fig. 4 is an incoherent image processing system that relates the input intensity $I_{i}(x, y)$ to the output intensity $I_{0}(x, y)$ by [21]

$$
\begin{aligned}
I_{i}(x, y) & =\iint \operatorname{ipsf}\left(x^{\prime}, y^{\prime}\right) I_{0}\left(x^{\prime}+x, y^{\prime}+y\right) \mathrm{d} x^{\prime} \mathrm{d} y^{\prime} \\
& =\operatorname{ipsf}(x, y) \otimes I_{0}(x, y),
\end{aligned}
$$

where $\otimes$ denotes 2 -D correlation defined by the integral in Eq. (6), and the ipsf is given by the absolute squared of the Fourier transform of the pupil function $p(x, y)$, i.e.,

$$
\operatorname{ipsf}(x, y)=|\mathfrak{F}\{p(x, y)\}|^{2}=\left|P\left(\frac{x}{\lambda f}, \frac{y}{\lambda f}\right)\right|^{2},
$$

where $\mathfrak{F}\{$.$\} denotes the Fourier transform operation,$ and we denote that $\mathfrak{F}\{p\}=P$, i.e., the uppercase function $P$, is the Fourier transform of the lowercase function $p . \lambda$ is the wavelength of the scanning laser light, and again $f$ is the focal length of the lens as shown in Fig. $\underline{4}$. It is interesting to point out that the correlation operation in Eq. (6) can be written in terms of convolution, because the ipsf, being the impulse ring according to our scheme, is symmetrical, and hence we can write

$$
I_{i}(x, y)=\operatorname{ipsf}(x, y) * I_{0}(x, y),
$$

which is the type of operation, i.e., convolution, shown in Eq. (1). Now, to code $I_{0}(x, y)$ according to our coding scheme discussed in Section 2, we need to realize a scanning impulse ring at the back focal plane of lens L. A CDG placed in the pupil plane of the scanning system would accomplish the realization. We shall now briefly discuss the grating in Section $\underline{4}$.

\section{Circular Dammann Grating}

A CDG is a phase grating that produces a set of uniform-intensity impulse rings at the focal plane [1]. When there is only one uniform-intensity impulse ring shown in the focal plane, we have a so-called first-order CDG [4]. The cross section of the firstorder CDG is shown in Fig. 5(a), where $T$ is the period of the grating. If the CDG is placed on the pupil plane in the scanning system of Fig. 4 , the focal plane intensity distribution is an impulse ring. Figure 5(b) shows the focal plane intensity distribution due to such a first-order CDG. The actual parameters of the grating and the optical system are $T=80 \mu \mathrm{m}$, $f=75 \mathrm{~cm}, \lambda=0.6328 \mu \mathrm{m}$, and the limiting circular aperture size of the grating is approximately $7 \mathrm{~mm}$ in diameter. We put a pinhole of approximately $0.038 \mathrm{~mm}$ diameter to sample the intensity pattern in the focal plane, i.e., we let $I_{0}(x, y) \approx \delta(x, y)$ to sample the intensity due to the grating placed in the front focal plane. In Fig. 5(b) we show the measured ring distribution. The radius of the ring [4] is given 


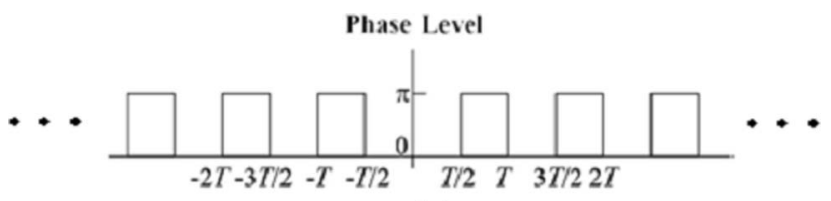

(a)

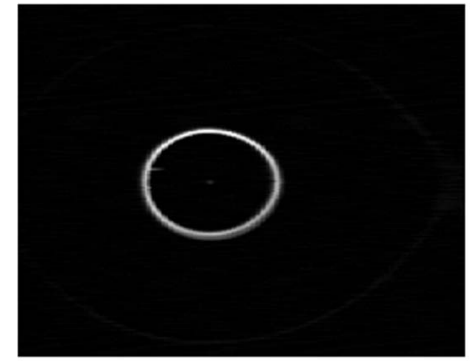

(b)

Fig. 5. (a) Cross section of a first-order Dammann grating and (b) an annular beam (focal plane intensity distribution due to the first-order CDG).

by $r_{0}=\lambda f / T \approx 5.6 \mathrm{~mm}$. The uniform-intensity impulse ring shown in Fig. 5(b) is called an annular beam, with which we scan the input image in two dimensions to optically code the image. In Section $\underline{5}$ we show some experimental results.

\section{Experimental Results}

The input object is a transparency of the letters "VT." The letters are approximately $4 \mathrm{~mm} \times 5 \mathrm{~mm}$ and are transmission on an opaque background. Figure 6(a) shows the scanned version of the transparency when it is scanned by a sharply focused Gaussian laser beam. When the object is scanned by the annular beam shown in Fig. 5(b), we have the coded output shown in Fig. 6(b). Note that we have designed the size of "VT" to be smaller than that of the scanning annular beam to minimize the circular bump as discussed in Section 2. Note also that the optical scanned results are basically identical to the coded image shown in Fig. 3(c) in computer simulation. Figure 6(c) shows the self-convolution of the annular beam, which can be compared with the simulations shown in Fig. 1(b). In Fig. 6(d), we show the cross section of Fig. 6(c) through the peak. And finally, the decoded image is shown in Fig. 6(e). This decoded image is done digitally according to Eq. (3), where the coding and decoding function is given by Fig. 5(b) with the coded image given by Fig. 6(b). Again, the result shown in Fig. 6(e) is basically identical to that of Fig. 3(d). If preferred, decoding can be done optically using the same optical system shown in Fig. 4. The coded image is simply inputted to a spatial light modulator that is subsequently scanned by the same annular beam used for coding.

\section{Inverse Filtering}

In Section $\underline{5}$, we provided experimental verification of the idea presented in Section 2. In Section 2, we observed that the quality of the decoded image depends on the size of the coding ring as evident from

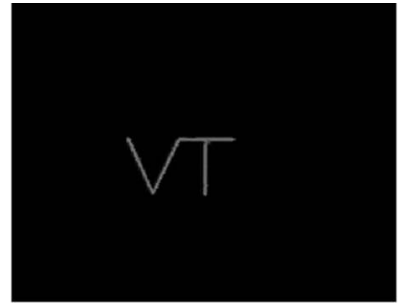

(a)

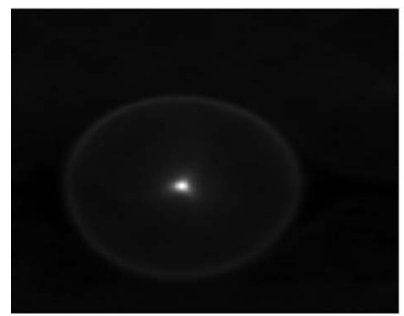

(c)

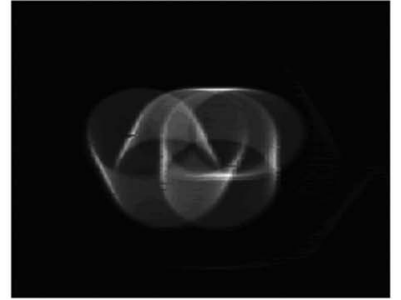

(b)

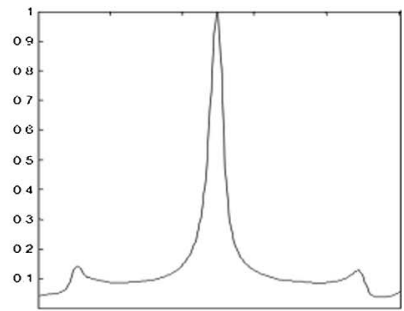

(d)

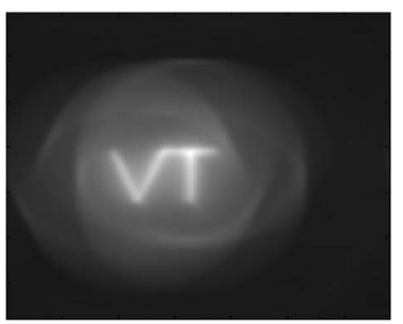

(e)

Fig. 6. (a) Original "VT" image, (b) optically coded image, (c) selfconvolution of the annular scanning beam, (d) cross section of (c) through the peak, (e) digitally decoded image.

comparing the results of Figs. 2(d) and 3(d). The results indicate that the coding ring should be larger than the object in order to avoid the effect of the bump from the psf of the overall system. However, the larger the ring, the greater the attenuation of information in the decoded image at high frequencies will be, as the transfer function of the overall system will go to zero at a lower spatial frequency when the radius of the ring increases. To see this explicitly, we assume the coding ring on the object's plane is an ideal impulse ring given by $\delta\left(r-r_{0}\right)$, where $r=\sqrt{x^{2}+y^{2}}$, and $r_{0}$ is the radius of the ring. According to Eq. (3), the overall system's psf is given by psf $=c(x, y) * d(x, y)=\delta\left(r-r_{0}\right) * \delta\left(r-r_{0}\right)$. The system's transfer function is, therefore, given by $H(\rho)=\mathfrak{F}\{\operatorname{psf}\}=\mathfrak{F}\left\{\delta\left(r-r_{0}\right) * \delta\left(r-r_{0}\right)\right\}$, where $\rho$ is the spatial frequency associated with the spatial coordinate $r$. Since $\mathfrak{F}\left\{\delta\left(r-r_{0}\right)\right\}=2 \pi r_{0} J_{0}\left(2 \pi r_{0} \rho\right)$, we find

$$
H(\rho)=\mathfrak{F}\left\{\delta\left(r-r_{0}\right)\right\} \mathfrak{F}\left\{\delta\left(r-r_{0}\right)\right\}=\left[2 \pi r_{0} J_{0}\left(2 \pi r_{0} \rho\right)\right]^{2}
$$

Assuming the first zero of the zero-order Bessel, $J_{0}$, is $z_{1}$, we therefore find that the spatial frequency at which it goes to zero is at $\rho=z_{1} / 2 \pi r_{0}$. Hence the larger the ring radius, the greater the attenuation of the 


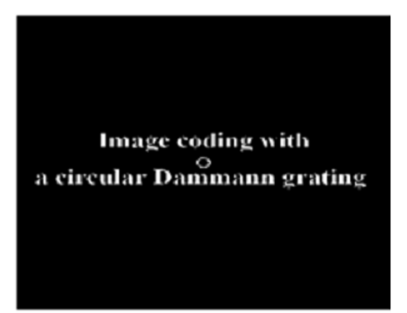

(a)

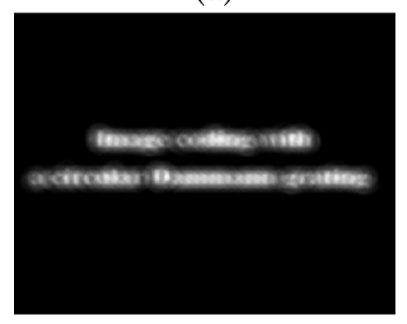

(c)

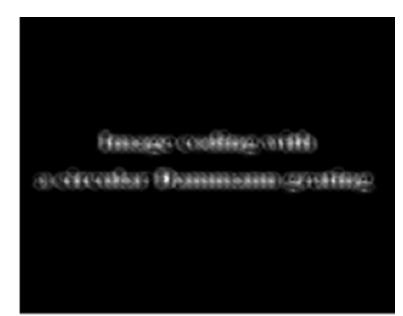

(b)

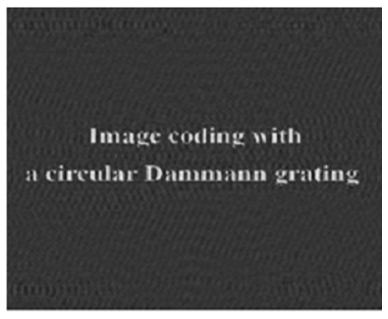

(d)
Fig. 7. (a) Original image, (b) coded image, (c) decoded image, (d) inverse filter of the coded image shown in (c).

information at higher frequencies will be. However, we can compensate for this attenuation by using simple inverse filtering:

$$
\begin{aligned}
& \text { inverse filtered decoded image } \\
& =\mathfrak{F}^{-1}\left\{\mathfrak{F}\left\{i_{d}\right\} /[H(\rho)+\varepsilon]\right\},
\end{aligned}
$$

where $\mathfrak{F}^{-1}$ denotes the inverse Fourier transform, and $\varepsilon$ is some constant. Since $H(\rho)$ is known, the implementation of the filter is straightforward. We have prepared a series of simulations to validate the effectiveness of the proposed inverse filter. Figure 7 shows the results when a small ring is used for coding. Figure 7(a) shows the original image. The coding ring is shown in the center of the figure to il-

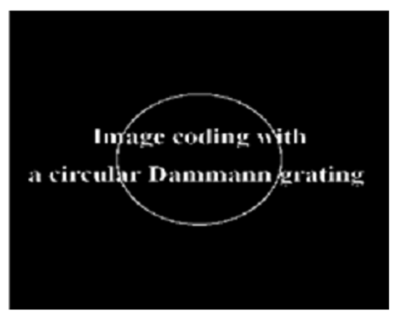

(a)

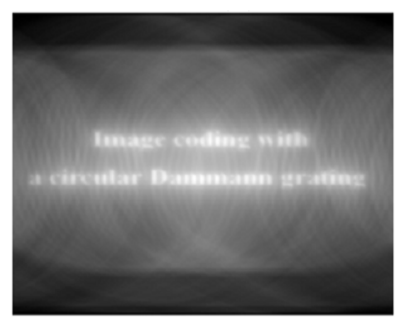

(c)

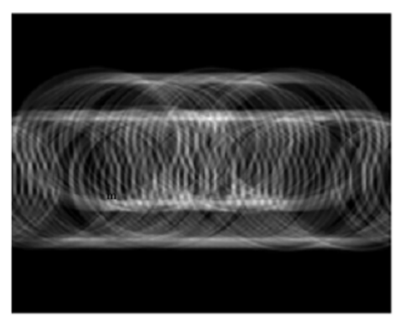

(b)

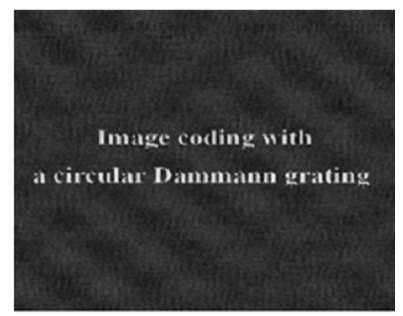

(d)
Fig. 8. (a) Original image, (b) coded image, (c) decoded image, (d) inverse filter of the coded image shown in (a).

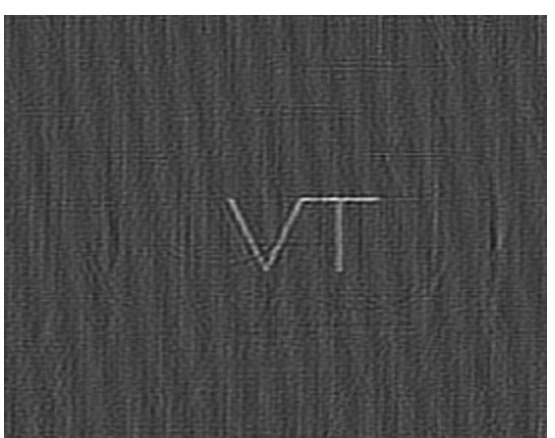

Fig. 9. Inverse filter of Fig. $\underline{6(\mathrm{e})}$.

lustrate the relative size of the ring and the text to be coded. Figure 7(b) is the coded image, and Fig. 7(c) is the decoded image. In this case, the size of the ring is small, as the ring would overlap any character of the text and hence the "bump effect" is obvious. Figure $7(\mathrm{~d})$ shows the result of inverse filtering. $\varepsilon$ is chosen to have a good visual effect on the text. Note that by no means do we try to optimize inverse filtering here. Figure 8 shows the results when a large ring is used. Finally, in Fig. 9, we show inverse filtering of the decoded image from Fig. 6(e). In this case, $H(\rho)$ is computed using the autoconvolution of the ring shown in Fig. 6(c). We see that the highfrequency contents of the decoded image have been restored, as the edges of the image have been sharpened.

\section{Concluding Remarks}

We proposed an optical coding system using optical scanning. The coding and decoding functions are of the form of an annular beam, which approximately satisfies the Delta reconstruction condition commonly used in aperture coded imaging for $\mathrm{x}$ ray or gamma rays. Computer simulation results have been verified by optical experiments. We want to point out that the resolution of the coding/decoding system depends on how good the approximation in Eq. (4) becomes after the autoconvolution of the annular beam, which in turn depends on how thin we can make the ring in Fig. 5(b). As it turns out, the thinness of the ring is $\propto \lambda f \overline{/ r_{0}}$ and the factor $f / r_{0}$ is reminiscent of the numerical aperture of a lens. We found that the proposed system is an efficient way of performing optical coding. While planar objects have been used in the optical experiments, it may be useful to further investigate this system for three-dimensional coding, which we plan to do.

This work was supported by the Korea Research Foundation (KRF) grant KRF-2008-313-D00763. We thank Shuai Zhao for his help with the use of the grating.

\section{References}

1. C. Zhou, J. Jia, and L. Liu, "Circular Dammann grating," Opt. Lett. 28, 2174-2176 (2003). 
2. H. M. Shang, S. L. Toh, Y. Fu, C. Quan, and C. J. Tay, "The use of circular optical grating for measuring angular rotation of mirrors," Opt. Lasers Eng. 36, 487-500 (2001).

3. M. Toda, "Single mode behavior of a circular grating for potential disk-shaped DFB lasers," IEEE J. Quantum Electron. 26, 473-481 (1990).

4. S. Zhao, J. F. Wen, and P. S. Chung, "Simple focal-length measurement technique with a circular Dammann grating," Appl. Opt. 46, 44-49 (2007).

5. G. A. Tyler, "Image coding with far-field holograms," Appl. Opt. 17, 2768-2772 (1978).

6. E. Klotz, U. Tiemens, and H. Weiss," X-ray tomography by grid coding," Appl. Opt. 19, 474-476 (1980).

7. G. R. Gindi, R. G. Paxman, and H. H. Barrett, "Reconstruction of an object from its coded image and object constraints," Appl. Opt. 23, 851-856 (1984).

8. N. M. Ceglio, D. T. Attwood, and E. V. George, "Zone-plate coded imaging of laser-produced plasma," J. Appl. Phys. 48, 1566-1669 (1977).

9. W. T. Dallas, "Prefolded Fourier filter reconstruction of coded aperture images," Opt. Acta 26, 1359-1365 (1979).

10. H. H. Barrett and F. A. Morrigan, "Fresnel zone plate imaging of gamma rays: theory," Appl. Opt. 12, 2686-2702 (1973).

11. E. Klotz, R. Linde, and H. Weiss," A new method for deconvoluting coded aperture images of 3-D x-rays objects," Opt. Commun. 12, 183-187 (1974).
12. G. Indebetouw and W. P. Sing, "Optical scanning reconstruction of Fresnel zone plate coded images," Appl. Opt. 20, 3996-3998 (1981).

13. G. Indebetouw, "Scanning heterodyne reconstruction of coded aperture images," Opt. Laser Technol. 18, 268-272 (1986).

14. R. C. Gonzalez and P. Wintz, Digital Image Processing (Addison-Wesley, 1977).

15. S. Lai and M. A. Neifeld, "Digital wavefront reconstruction and its applications to image encryption," Opt. Commun. 178, 283-289 (2000).

16. B. Wang, C.-C. Sun, W.-C. Su, and A. Chiou, "Shift-tolerance property of an optical double-random phase-encoding encryption system,” Appl. Opt. 39, 4788-4793 (2000).

17. B. Zhu, S. Liu, and Q. Ran, "Optical image encryption based on multifractional Fourier transforms," Opt. Lett. 25, 1159-1161 (2000).

18. P. C. Magensen and J. Gluckstad, "Phase-only optical decryption of a fixed mask," Appl. Opt. 40, 1226-1235 (2001).

19. E. Tajahuerce, J. Lancis, B. Javidi, and P. Andres, "Optical security and encryption with totally incoherent light," Opt. Lett. 26, 678-680 (2001).

20. T.-C. Poon, T. Kim, and K. Doh, "Optical scanning cryptography for secure wireless transmission," Appl. Opt. 42, 6496-6503 (2003).

21. T.-C. Poon, Optical Scanning Holography with MATLAB (Springer, 2007). 